\title{
Cryogenics for the MuCool Test Area (MTA)
}

\author{
Christine Darve, Barry Norris, Liujin Pei \\ Fermilab, Cryogenics department, MS347 \\ Batavia, Illinois, 60510
}

\begin{abstract}
MuCool Test Area (MTA) is a complex of buildings at Fermi National Accelerator Laboratory, which are dedicated to operate components of a cooling cell to be used for Muon Collider and Neutrino Factory R\&D. The long-term goal of this facility is to test ionization cooling principles by operating a 25-liter liquid hydrogen $\left(\mathrm{LH}_{2}\right)$ absorber embedded in a 5 Tesla superconducting solenoid magnet. The MTA solenoid magnet will be used with RF cavities exposed to a high intensity beam. Cryogens used at the MTA include $\mathrm{LHe}, \mathrm{LN}_{2}$ and $\mathrm{LH}_{2}$. The latter dictates stringent system design for hazardous locations. The cryogenic plant is a modified Tevatron refrigerator based on the Claude cycle. The implementation of an in-house refrigerator system and two 300 kilowatt screw compressors is under development. The helium refrigeration capacity is $500 \mathrm{~W}$ at $14 \mathrm{~K}$. In addition the MTA solenoid magnet will be batch-filled with LHe every 2 days using the same cryo-plant. This paper reviews cryogenic systems used to support the Muon Collider and Neutrino Factory R\&D programs and emphasizes the feasibility of handling cryogenic equipment at MTA in a safe manner.
\end{abstract}

Keywords: Muon cooling, cryogenics, hydrogen absorbers, helium plant

PACS: 07.20.Mc

\section{INTRODUCTION}

Cooling channels are fundamental components for the development of a future Muon Collider or Neutrino Factory [1]. Because muons decay very quickly, ionization cooling appears as the preferred principle to reduce the normalized emittance of muon beams. These arguments drive the choice of a low- $\mathrm{Z}$ medium in order to reduce the Coulomb multiple scattering and maximize the ionization energy loss. The optimal candidates are thus hydrogen (as a fluid) and beryllium or aluminum (as metals). High gradient RF cavities are necessary to restore the beam's longitudinal momentum after cooling. The choices made in each step, cooling and reacceleration, will affect the final quality of the beam that can be achieved.

The cryo-engineering aspect of this research must address several challenges. The first is to design a test facility according to Fermilab hydrogen safety standards while housing absorbers filled with $\mathrm{LH}_{2}$ contained in a manifold closed by two extremely thin aluminum windows [2]. Another technical challenge is the insertion of a $\mathrm{LH}_{2}$ absorber into a high-magnetic field at the proximity of RF cavities without violating electrical safety standards.

In the present paper, we review the characteristics of the temporary and future MTA cryogenic systems to support MuCool research. 


\section{USER REQUIREMENTS AND CRYOGENIC FACILITIES}

In terms of cryogenic fluid requirements, MTA facilities provide hydrogen, helium and nitrogen to the users. One of the purposes of the MTA building complex is to test two types of $\mathrm{LH}_{2}$ absorbers: a convection-style one and a forced-flow one. In both cases, the ultimate goal of the testing is to deposit the maximum heat load to the $\mathrm{LH}_{2}$ absorber volume, while limiting $\mathrm{LH}_{2}$ density fluctuations in the absorber volume. The MTA test facility will also provide cooling to a 5 Tesla NbTi superconducting solenoid magnet.

\section{Operations based on Dewar fed LHe system}

The mechanical, electrical and control approaches of this project were executed according to Fermilab design and review procedures. Hydrogen gas is stored in a separate building. An outdoor $21 \mathrm{~m}^{3}$ vacuum buffer tank is connected to the cryostat vacuum vessel. The function of this vacuum buffer tank is to collect gas expanded to standard temperature and pressure in case of rupture from the hydrogen volume to the vacuum volume. This absorber was designed and built by KEK to validate the International Muon Ionization Cooling Experiment (MICE) absorber design [3]. The $\mathrm{LH}_{2}$ absorber was instrumented with platinum-cobalt type temperature sensors and contained in a cryostat before being shipped to Fermilab. The beam energy deposition is simulated by warm helium flowing through a coil located inside of the $\mathrm{LH}_{2}$ absorber volume. This dynamic heat load is transferred from the $\mathrm{LH}_{2}$ to the cold helium flow through a manifold-integrated heat exchanger by means of convection heat transfer. Numerical simulations support the assumption that up to $100 \mathrm{~W}$ can be extracted from the $\mathrm{LH}_{2}$ absorber by natural convection heat transfer. User requirements were to integrate the KEK cryostat in a system to operate at $\mathrm{LH}_{2}$ conditions. Because the final refrigeration system is not operational yet, cold helium was transferred from a 500 liter LHe Dewar located outside of the experimental hall to the KEK cryostat through a 25-meter long transfer line. This first run has validated the feasibility of MTA control processes, instrumentation and safety equipments. Following the commissioning of the test, twenty different test points (different $\mathrm{LH}_{2}$ temperature and pressure conditions) were investigated. Results are reported together with reflections to upgrade both the cryo-system and KEK absorber [2] [4-5].

Similar cryogenic installation is under construction to feed the superconducting solenoid magnet. The solenoid magnet liquid helium and nitrogen vessels are refilled every 2 days from the Dewar located outdoors.

\section{A future continuous helium supply}

Work is presently underway to install the required refrigeration system for longterm MuCool research. User requirements are to design the cryostat and cryo-loop integrating the forced-flow $\mathrm{LH}_{2}$ absorber in the bore of the superconducting solenoid magnet and, simultaneously, to provide cryogenic facilities capable of delivering appropriate refrigeration. A continuous flow of $27 \mathrm{~g} / \mathrm{s}, 14 \mathrm{~K}$ helium is necessary to cool the 25 liters of sub-cooled $\mathrm{LH}_{2}$ flowing in a closed loop composed of a 6.9-liter 
absorber, a $\mathrm{LH}_{2}$ pump, a He/ $\mathrm{H}_{2}$ counter-flow heat exchanger and a heater. Although a conceptual design for the forced-flow system was completed in 2002 [6-7] an alternative solution using the existing cryostat from the SAMPLE experiment is being considered [8]. Numerical simulations were necessary to size the cryo-system and to optimize the $\mathrm{LH}_{2}$ flow hydro-dynamics [7] so that up to $300 \mathrm{~W}$ can be deposited by the dummy beam to the $\mathrm{LH}_{2}$ absorber.

A refrigerator and compressor system are being installed to fulfill this specification. The compressor room houses rotating machinery, i.e. two (2) Sullair 300 kilowatt 2stage oil injected screw compressors with associated after-coolers and coalescing filters. Each compressor is hooked up to a purification skid to remove oil vapor contamination from helium gas. Each purification skid includes a coalescing filter, a charcoal adsorber, and a final filter. The compressor system is connected to a Tevatron satellite refrigerator style, which was modified to match the MTA requirements. One helium and one nitrogen storage tank are located outdoors. A transfer line connects the refrigerator complex to the experimental hall and will be used to feed the superconducting solenoid magnet and the forced-flow cryostat.

The full capacity of the MTA future refrigerator complex will permit two operational temperature ranges: $14 \mathrm{~K}$ to support the forced-flow $\mathrm{LH}_{2}$ absorber system and $5 \mathrm{~K}$ to "batch fill" the superconducting solenoid magnet. Therefore the cryo-plant design approach is to allow the refrigeration system to switch from $14 \mathrm{~K}$ to $5 \mathrm{~K}$ mode. Figure 1 shows the flow schematic of the MTA refrigeration system.

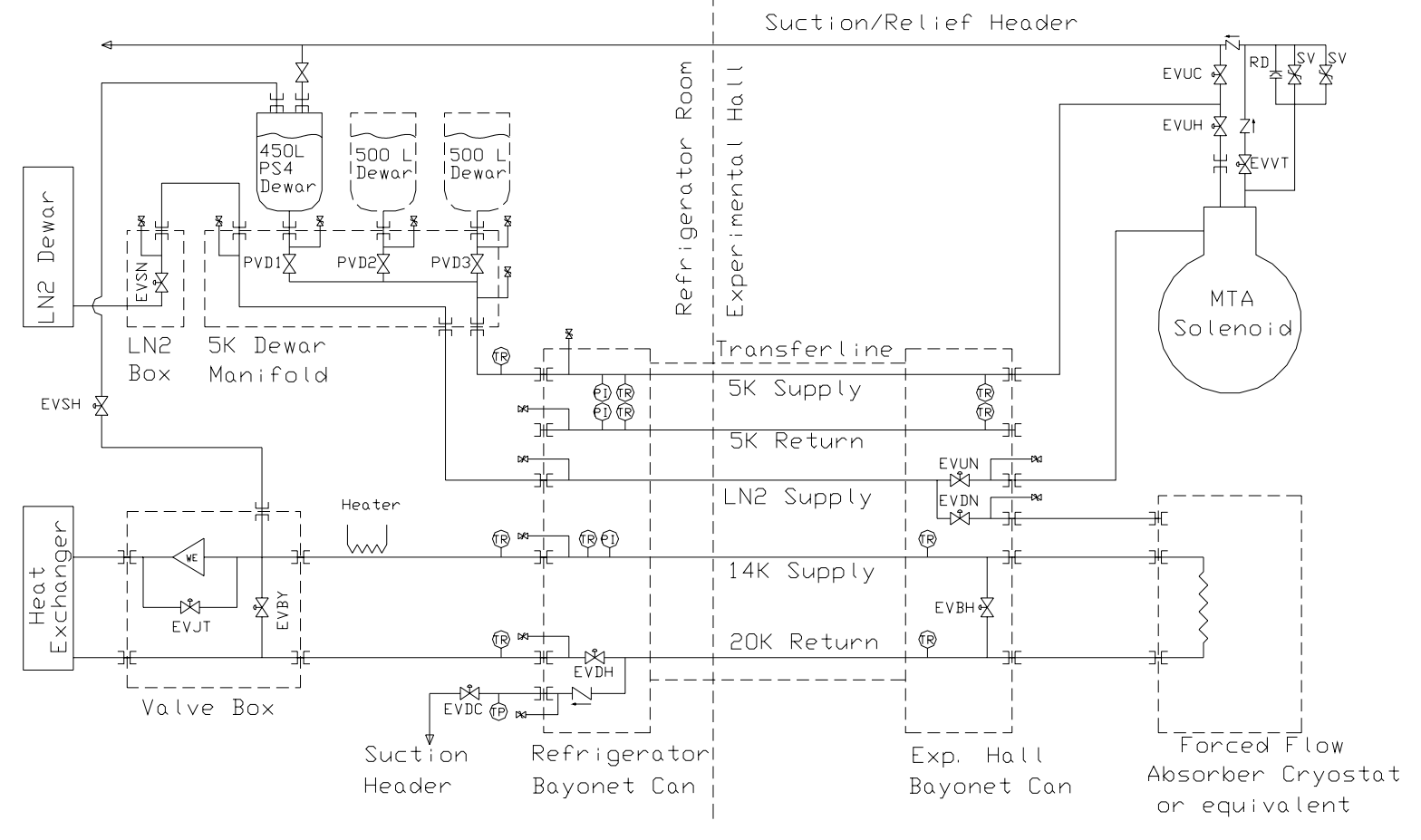

Figure 1. Overall scheme of MTA refrigeration including support of the $5 \mathrm{~K}$ helium needs for the superconducting solenoid magnet and the $14 \mathrm{~K}$ system for absorber experiments. 


\section{ELECTRICAL COMPONENT SOLUTIONS}

The design of the different cryo-systems (forced-flow $\mathrm{LH}_{2}$ absorber, KEK $\mathrm{LH}_{2}$ absorber and superconducting solenoid magnet) utilizes the expertise of existing US hydrogen experiments [8-9] and Fermilab $\mathrm{LH}_{2}$ target guidelines. Due to the presence of hydrogen in the experimental hall, all ignition sources must be rated Class I (areas with flammable vapors), Division 2 (hazard normally not present), group B (hazard is hydrogen) in accordance with the National Electrical Code (NEC). If these conditions cannot be met then devices must be engineered intrinsically safe or placed in a shunt trip circuit. For this purpose a nitrogen purge box is located in the experimental hall to house non-code devices and electronics, which are in proximity of devices located inside the $\mathrm{LH}_{2}$ absorbers. For instance, Cernox ${ }^{\circledR}$, platinum-cobalt, platinum and carbon temperature sensors are excited from electronics located in the experimental hall purged box. Cernox ${ }^{\circledR}$ is the temperature sensor of choice due to its reliable performances at cryogenic temperatures and under magnetic field. Hydrogen safety requirements also drive the use of diffusion pump, capacitance-type vacuum gauges, MC type cable and PLTC cables. The experimental hall ventilation system is monitored by a flow switch mounted at the end of the ventilation duct, which would become a hazardous area in the presence of hydrogen in the experimental hall. Therefore, an intrinsically safe barrier is required to connect the electrical cable to the I/O box providing read-out. Interlocks set the system in fail-safe mode if hydrogen or helium were to leak in the experimental hall. Further, flammable gas detectors, ODH detectors and purged-system pressure transducers are continuously monitoring the MTA building conditions through the QUADLOG ${ }^{\circledR}$ safety PLC. Alarms are activated if the read-out is beyond the chosen set values. Depending on the degree of emergency, audio and visual warnings, automatic fluid venting, or activation of the FIRUS system (Fermilab Fire Department is alerted) can occur. Additional engineering solutions were considered throughout the MTA projects demanding developments [5].

Hydrogen safety issues can trigger a broad variety of answers [8-10]. For instance, the NFPA panel is delegated to generate $\mathrm{H}_{2}$ standards and create a Proposed Hydrogen Technology Correlating Committee. Although safety standards vary according to laboratory policy, many guidelines exist to run a safe but realistic hydrogen project.

\section{PROCESS AND CONTROL SYSTEM SOLUTIONS}

Two types of programmable logic controller (PLC) technology are used for the operation of $\mathrm{LH}_{2}$ absorber and superconducting solenoid magnet. In the case of the superconducting solenoid magnet operation, the data acquisition (DAQ) and control system are relaxed from explosion proof criteria and a conventional PLC was implemented to automatically supply helium and nitrogen to the solenoid magnet. The implementation of the instrumentation and controls for the MTA helium cryo-plant in the compressor and refrigerator rooms are based on Tevatron standards.

Finally, the data-acquisition system and control must accurately monitor a hazardous environment like flammable gas, and oxygen deficiency hazard. The Fermilab safety committee requires the use of a safety PLC when operating a test with 
hydrogen presence. Siemens-Moore QUADLOG ${ }^{\circledR}$ safety PLC is designed for this type of application and permits us to operate in a safe manner using dual architecture. QUADLOG ${ }^{\circledR}$ makes use of device redundancy. It provides a very reliable system with the fault tolerance and special self-testing software. QUADLOG® safety PLC is used for continuous operations or batch processing and is fail-safe. QUADLOG ${ }^{\circledR}$ is a programmable control system designed specifically for critical applications, such as emergency shutdown systems, fire and gas detection systems. QUADLOG ${ }^{\circledR}$ combines the beneficial features of a PLC (such as modularity, ladder logic and sequential programming) with high safety, high availability, and extensive diagnostics. It also incorporates continuous PID control, analog $\mathrm{I} / \mathrm{O}$, and a variety of operator interface options not typically available from a PLC.

\section{CONCLUDING COMMENTS}

The Fermilab MTA facility is an operational hydrogen facility equipped with cryogenic capacity. The cryogenic utilities and developments supporting the MuCool research were described. The MTA was fully designed to comply with hydrogen project guidance. A first $\mathrm{LH}_{2}$ absorber was successfully tested using a temporary liquid helium supply system. This first hydrogen test permitted a validation of the reliability of the process system operating under a hazardous environment.

\section{ACKNOWLEDGMENTS}

The authors want to thank the Cryogenic Department for the donation of equipment and for its technical availability to support the work. Fermilab is operated by Universities Research Association Inc. under contract No. DE-AC02-76CH03000 with the U. S. Department of Energy.

\section{REFERENCES}

1. D. Kaplan et. al., "Muon-cooling research and development", Nucl. Instr. and Meth, 241-248 (2004).

2. M. Cummings, "Progress in the Liquid Hydrogen Absorber for the MICE Cooling Channel", submitted to PAC’05 proceeding (2005).

3. S. Ishimoto et. al., "Convection-type $\mathrm{LH}_{2}$ absorber R\&D for muon ionization cooling”, Nucl. Instr. and Meth. A 503, 2003, pp. 396.

4. B. Norris et. Al, "Reflections on Initial MTA Tests”, FNAL/Cryogenic Dept. Iinternal Note (2004).

5. A. Bross et. al, “An upgrade for the MuCool Test Area”, submitted to CEC'05 proceeding (2005).

6. C. Darve et al., "Cryogenic Design for a Liquid Hydrogen Absorber System”, Proceeding of ICEC19, edited by G. Gistau Baguer and P.Seyfert, Narosa, Grenoble, France, 2002, pp. 593-596.

7. C. Darve et al., "The Liquid Hydrogen System for the MuCool Test Area," in Advances in Cryogenic Engineering 49A, edited by J. Waynert et al., Plenum, New York, 2003, pp. 48-55.

8. E.J. Beise et al., “A high power liquid hydrogen target for parity violation experiments”, Nucl. Instr.and Meth. A378, 1996, pp. 383-391.

9. J. G. Weisend II et. al, "Safety Aspects Of The E158 Liquid Hydrogen Target System”, Proceeding of ICEC19, edited by G. Gistau Baguer and P.Seyfert, Narosa, Grenoble, France, 2002, pp. 605-608.

10. Mike Green, "Hydrogen safety issues compared to safety issues with methane and propane", submitted to CEC’05 proceeding (2005). 